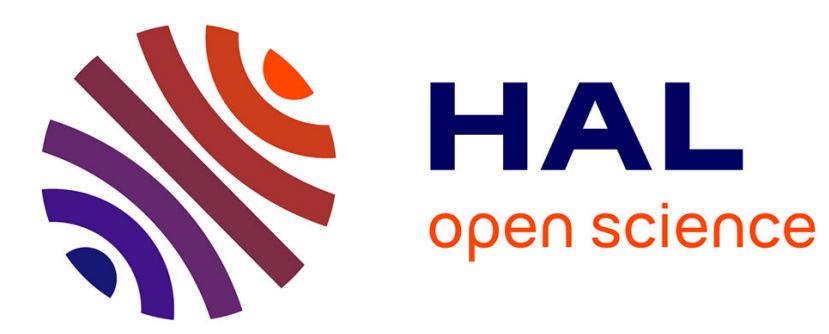

\title{
Doppler Estimation and Data detection for Underwater Acoustic ZF-OFDM Receiver
}

Alain Kibangou, Laurent Ros, Cyrille Siclet

\section{To cite this version:}

Alain Kibangou, Laurent Ros, Cyrille Siclet. Doppler Estimation and Data detection for Underwater Acoustic ZF-OFDM Receiver. ISWCS 2010 - 7th International Symposium on Wireless Communication Systems, Sep 2010, York, United Kingdom. pp.n.c. hal-00498161

\section{HAL Id: hal-00498161 \\ https://hal.science/hal-00498161}

Submitted on 6 Jul 2010

HAL is a multi-disciplinary open access archive for the deposit and dissemination of scientific research documents, whether they are published or not. The documents may come from teaching and research institutions in France or abroad, or from public or private research centers.
L'archive ouverte pluridisciplinaire HAL, est destinée au dépôt et à la diffusion de documents scientifiques de niveau recherche, publiés ou non, émanant des établissements d'enseignement et de recherche français ou étrangers, des laboratoires publics ou privés. 


\title{
Doppler Estimation and Data detection for Underwater Acoustic ZF-OFDM Receiver
}

\author{
Alain Y. Kibangou ${ }^{* 1}$, Laurent Ros ${ }^{\# 2}$, Cyrille Siclet ${ }^{\# 3}$ \\ * Control Systems Department, GIPSA-Lab, University Joseph Fourier, CNRS \\ BP 46, F-38402 Grenoble Cedex, France \\ ${ }^{1}$ alain.kibangou@ujf-grenoble.fr \\ \# Image and Signal Department, GIPSA-Lab, University Joseph Fourier, Grenoble-INP \\ BP 46, F-38402 Grenoble Cedex, France \\ 2 laurent.ros@gipsa-lab.grenoble-inp.fr \\ ${ }^{3}$ cyrille.siclet@gipsa-lab.grenoble-inp.fr
}

\begin{abstract}
A new scheme for Doppler estimation and data detection for OFDM underwater acoustic communications is derived in this paper. We design the OFDM symbol as a concatenation of two sub-blocks. The first one is shorter and carries out few data with few subcarriers whereas the second one contains both informative and pilot symbols. The first sub-block serves to estimate the Doppler scaling factor while the pilots in the second sub-block are used for estimating the equivalent channel. Both estimation approaches are based on high resolution methods for solving harmonic retrieval problems in time and frequency domains respectively. Each sub-block contains a cyclic suffix, which allows having more data to be processed. The effectiveness of the proposed scheme is evaluated by means of simulation results.
\end{abstract}

\section{INTRODUCTION}

Underwater acoustic channels are typically wideband in nature due to the small ratio of carrier frequency to the signal bandwidth, which introduces frequency-dependent Doppler shifts [1]. They also exhibit several propagation paths, so that the received signal is equivalent to the sum of several signals with different amplitude and time delays. Multipath delay and Doppler effects constitute the main obstacles to robust underwater acoustic communication. One common approximation is to treat the channel as having a common Doppler scaling factor on all propagation paths.

Orthogonal Frequency Division Multiplexed (OFDM) signals are particularly attractive because they lead to relatively simple channel estimation and data detection algorithms. Indeed, since subcarrier only experiences flat fading, complex time-domain equalizers are not necessary [2]. However, OFDM signals are particularly vulnerable to Doppler effects that destroy the orthogonality between subcarriers and thus induce intercarrier interference (ICI). In order to adequately recover the transmitted information, algorithms at the receiver must include estimation and compensation of the Doppler scaling factor, channel estimation, and information symbols estimation.

For estimating the Doppler scaling factor, several approaches have been suggested in the literature (see [3] for example). In the OFDM case, they are based on the use of preamble and postamble of a packet consisting of multiple
OFDM blocks [1] or by exploiting correlation induced by the cyclic prefix [4]. Then, the received signal is resampled by using a sampling period related to the estimated Doppler scaling factor. Recently, the authors introduced a new scheme for estimating both Doppler and channel parameters by solving harmonic retrieval problems [5]. In this paper, we propose an improved version of the previous algorithm with a better use of the bandwidth and without explicitly estimating the paths gain and delay. Indeed, the lack of robustness to noise of the channel parameter estimators was a major drawback of the approach in [5], [6]. Herein, instead of using a zero padding scheme, we introduce a cyclic suffix in the OFDM signal design. As in [5], the received data are processed block-byblock. The advantage of the proposed scheme is to avoid data resampling and residual CFO estimation and compensation.

\section{SYSTEM MODEL}

We consider an OFDM transmission system where each OFDM block is constituted with two sub-blocks of respective length $T_{u}$ and $T_{d}$, with $T_{d} / T_{u} \in \mathbb{N}$, as depicted in Fig. 1. Each sub-block is followed by a cyclic suffix (CS) of duration $T_{g}$. Therefore, the overall OFDM block duration is $T=T_{u}+T_{d}+2 T_{g}$.

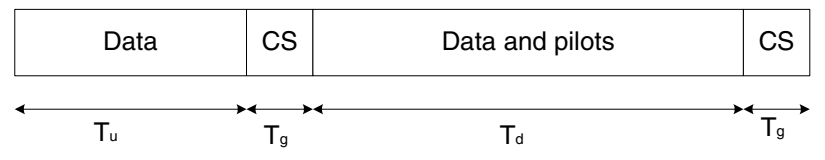

Fig. 1. Structure of the OFDM block

We denote by $c_{q}$ the data symbols in the first sub-block. They are all informative and thus unknown to the receiver. In the second sub-block, all data symbols $d_{k}$ are not informative; some of them are known to the receiver and will serve to estimate the equivalent channel parameters. In baseband, the signal $s($.$) to be transmitted can be viewed as a mixture of two$ signals $s_{u}($.$) and s_{d}($.$) , each one corresponding respectively$ to the first and to the second sub-blocks:

$$
s(t)=s_{u}(t)+s_{d}(t)
$$


with

$$
\begin{aligned}
& s_{u}(t)=\eta \sum_{q \in \mathcal{K}_{u}} c_{q} e^{j 2 \pi q Q \Delta f t} \Pi_{T_{u}+T_{g}}(t) \\
& s_{d}(t)=\sum_{k \in \mathcal{K}_{d}} d_{k} e^{j 2 \pi k \Delta f t} \Pi_{T_{d}+T_{g}}\left(t-T_{u}-T_{g}\right)
\end{aligned}
$$

where $\Delta f=1 / T_{d}$ denotes the minimal frequency spacing between consecutive subcarriers, $\Pi_{T}$ allows taking the CS operation into account, i.e. $\Pi_{T}(t)=1, t \in[0, T]$, and $\Pi_{T}(t)=0$, otherwise, $\eta$ allows controlling the power of the first sub-block so that both sub-blocks have the same power, $Q$ is an integer that allows increasing the frequency spacing between the subcarriers in $s_{u}(),. \mathcal{K}_{u}$ (resp. $\mathcal{K}_{d}$ ) the set of subcarriers in the first (resp. second) sub-block.

In the sequel, we consider that $\mathcal{K}_{u} \subset \mathcal{K}_{d}$, meaning that some of the subcarriers of the second sub-block are reused in the first sub-block. By denoting $K_{u}$ (resp. $K_{d}$ ) the cardinality of $\mathcal{K}_{u}$ (resp. $\mathcal{K}_{d}$ ), i.e. the number of used subcarriers, we get $K_{u}<K_{d}, \mathcal{K}_{d}=\left\{-K_{d} / 2, \cdots, K_{d} / 2+1\right\}$, and $\mathcal{K}_{u}=\left\{q_{0}, \cdots, q_{0}+K_{u}-1\right\}$, for a given integer $q_{0}$.

In passband, the analytical representation of the signal to be transmitted is $x(t)=s_{u}(t) e^{j 2 \pi f_{c} t}+s_{d}(t) e^{j 2 \pi f_{c} t}, t \in[0, T]$, with $f_{c}$ the central carrier frequency. This signal is transmitted through a multipath underwater channel whose impulse response is given by:

$$
h(t, \tau)=\sum_{p=1}^{P} A_{p}(t) \delta\left(\tau-\tau_{p}(t)\right),
$$

where $A_{p}(t)$ and $\tau_{p}(t)$ are respectively the gain and the delay associated with the $p$ th path. The following assumptions are adopted:

- All paths are affected by a similar Doppler scaling factor $a$ such that $\tau_{p}(t)=\tau_{p}-a t$.

- The path delays $\tau_{p}$, the gains $A_{p}$, and the Doppler scaling factor $a$ are constant over the block duration $T$.

- The guard interval $T_{g}$ is chosen so that $T_{g}>\max \left\{\tau_{p}\right\}=$ $\tau_{\max }$.

Therefore, the received signal results on a mixture of scaled, dilated or compressed, and delayed versions of the original OFDM block as shown in Fig. 2. Hence, we can define two

$\mathrm{I}_{1}$

\begin{tabular}{|l|l|l|l|}
\hline \\
\hline
\end{tabular}

Fig. 2. Multipath and Doppler effect on the OFDM block time windows $I_{1}$ and $I_{2}$ where the received signal strictly depends on the first and second sub-blocks respectively. These time windows are defined by: $I_{1}=\left[\frac{\tau_{\max }}{\lambda}, \frac{T_{u}+T_{g}+\tau_{\min }}{\lambda}\right]$ and $I_{2}=\left[\frac{T_{u}+T_{g}+\tau_{\max }}{\lambda}, \frac{T+\tau_{\min }}{\lambda}\right]$, where $\tau_{\min } \leq \tau_{p} \leq \tau_{\max }, p=$ $1, \cdots, P$, and $\lambda=1+a$.

In $I_{1}$ and $I_{2}$, the analytical representation of the received signal is respectively given as follows:

$$
\begin{aligned}
& y(t)=\eta \sum_{q=q_{0}}^{K_{u}+q_{0}-1} B_{q} c_{q} e^{j 2 \pi(1+a) \varphi_{q} t}+w(t), \quad t \in I_{1}(2) \\
& y(t)=\sum_{k=-K_{d} / 2}^{K_{d} / 2-1} B_{k} d_{k} e^{j 2 \pi(1+a) f_{k} t}+w(t), \quad t \in I_{2} \quad(3)
\end{aligned}
$$

where $\varphi_{q}=f_{c}+q Q \Delta f, f_{k}=f_{c}+k \Delta f, w(t)$ denoting the additive noise, $B_{q}=\sum_{p=1}^{P} A_{p} e^{-j 2 \pi \varphi_{q} \tau_{p}}$, and

$$
B_{k}=\sum_{p=1}^{P} A_{p} e^{-j 2 \pi f_{k} \tau_{p}}
$$

In both $I_{1}$ and $I_{2}$, the received signal $y($.$) can be respec-$ tively viewed as a mixture of $K_{u}$ and $K_{d}$ harmonics with constant magnitudes. In the sequel, we intend to solve the problem of Doppler scale factor estimation as a Harmonic retrieval one. We make use of these two time windows for first estimating the Doppler scaling factor and then recovering the informative symbols contained in the OFDM block. Since the time windows involved in the estimation process depend on $\lambda$, we assume that $\lambda$ is bounded, such that $\lambda_{\min } \leq \lambda \leq \lambda_{\max }$. One can note that $\lambda_{\max }$ and $\lambda_{\min }$ are related to the maximal and minimal velocities of the underwater vehicles, which can be a priori known. In addition, we assume that $\tau_{\max }$ is known and $\tau_{\min }=0$. Therefore the involved time windows are modified as follows

$$
I_{1}=\left[\frac{\tau_{\max }}{\lambda_{\min }}, \frac{T_{u}+T_{g}}{\lambda_{\max }}\right] \quad I_{2}=\left[\frac{T_{u}+T_{g}+\tau_{\max }}{\lambda_{\min }}, \frac{T}{\lambda_{\max }}\right] .
$$

Owing to the CS, the time windows to be used are larger than those obtained when using a ZP scheme as in [6]. Obviously, with more observations, an improvement of the resulting estimation method is expected.

\section{DOPPLER ESTIMATION}

The harmonic retrieval problem has been extensively studied in the literature (see [7], [8], [9], [10] for example). In general the magnitude are assumed to be constant as in the case for $y($.$) in I_{1}$ and $I_{2}$. For solving (2) as a harmonic retrieval problem, we make use of the high resolution method called HTLS (Hankel Total Least Squares) [11]. This algorithm can be viewed as a special case of the ESPRIT algorithm [9] and also as a Total Least Squares variant of Kung et al.'s algorithm [12]. Resorting to a high resolution method is mandatory since the Doppler is to be estimated from a very slight deviation of the actual subcarrier frequencies. A search 
on discretized space of frequencies could need a very short step of discretization, implying a huge complexity. In other hand, FFT based methods have a lower bound of resolution incompatible in such a situation.

In the noiseless case, we consider the $N$ samples $y_{n}, n=$ $n_{0}, \cdots, N+n_{0}-1$, of the first portion (2) of the received signal:

$$
y_{n}=\eta \sum_{q=q_{0}}^{K_{u}+q_{0}-1} c_{q} B_{q} e^{j 2 \pi \varphi_{q}(1+a) n T_{e}}=\eta \sum_{q=q_{0}}^{K_{u}+q_{0}-1} c_{q} B_{q} \phi_{q}^{n},
$$

$T_{s}$ being the sampling period. In a harmonic retrieval problem, we aim to estimate both the poles $\phi_{q}=e^{j 2 \pi \varphi_{q}(1+a) T_{s}}$ and the magnitudes $\eta c_{q} B_{q}$ of the $K_{u}$ harmonics.

For this purpose, let us first build with the samples $y_{n}$ the following $L \times M$ Hankel matrix

$$
\mathbf{Y}=\left(\begin{array}{ccccc}
y_{n_{0}} & y_{n_{0}+1} & y_{n_{0}+2} & \cdots & y_{n_{0}+M-1} \\
y_{n_{0}+1} & y_{n_{0}+2} & . \cdot & \cdots & y_{n_{0}+M} \\
y_{n_{0}+2} & . \cdot & . \cdot & \cdots & y_{n_{0}+M+1} \\
\vdots & . \cdot & . \cdot & \vdots & \vdots \\
y_{n_{0}+L-1} & y_{n_{0}+L} & \cdots & \cdots & y_{n_{0}+N-1}
\end{array}\right),
$$

with $N=L+M-1, L>K_{u}, M \geq K_{u}$. It admits the Vandermonde decomposition:

$$
\mathbf{Y}=\mathbf{S}_{1} \operatorname{diag}(\boldsymbol{\alpha}) \mathbf{T}_{1}^{T},
$$

where $\mathbf{S}_{1}$ and $\mathbf{T}_{1}$ are two Vandermonde matrices with $\phi_{q}$ as generators:

$$
\begin{aligned}
\mathbf{S}_{1} & =\left(\begin{array}{ccc}
1 & \cdots & 1 \\
\phi_{q_{0}} & \cdots & \phi_{K_{u}+q_{0}-1} \\
\vdots & \ddots & \vdots \\
\phi_{q_{0}}^{L-1} & \cdots & \phi_{K_{u}+q_{0}-1}^{L-1}
\end{array}\right) \in \mathbb{C}^{L \times K_{u}} \\
\mathbf{T}_{1} & =\left(\begin{array}{ccc}
1 & \cdots & 1 \\
\phi_{q_{0}} & \cdots & \phi_{K_{u}+q_{0}-1} \\
\vdots & \ddots & \vdots \\
\phi_{q_{0}}^{M-1} & \cdots & \phi_{K_{u}+q_{0}-1}^{M-1}
\end{array}\right) \in \mathbb{C}^{M \times K_{u}},
\end{aligned}
$$

while $\alpha$ contains the complex magnitudes of the $K_{u}$ harmonics:

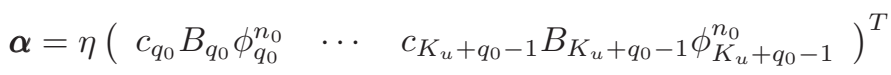

We intend to estimate the Doppler scaling factor from the angle of the generators $\phi_{q}$. In order to avoid any ambiguity since angles are obtained up to $2 \pi$, the sampling period can be selected such that the angle of the generators belongs to $[-\pi, \pi]$. For this purpose, it is enough to select $T_{s}$ such that:

$$
T_{s} \leq \frac{1}{2 \lambda_{\max } \max \left\{\varphi_{q}\right\}} .
$$

It should be noted that this condition corresponds precisely to the Nyquist-Shannon sampling theorem condition applied to the Doppler scaled spectrum of the received signal. We can also derive the following proposition:
Proposition 1: If the sampling period is such that $T_{s} \leq$ $\frac{1}{2 \lambda_{\max } \max \left\{\varphi_{q}\right\}}$, the Vandermonde matrices $\mathbf{S}_{1} \in \mathbb{C}^{L \times K_{u}}$ and $\mathbf{T}_{1} \in \mathbb{C}^{M \times K_{u}}, L>K_{u}, M \geq K_{u}$, with $\phi_{q}=e^{j 2 \pi \varphi_{q}(1+a) T_{s}}$ as generators are full column rank.

Proof: The Vandermonde matrices loose their rank if at least two generators have the same angle modulo $2 \pi$. Owing to condition (8) on the sampling period we know that the angles of the generators belong to $[0, \pi]$. Therefore, the angle of the generator are all distinct modulo $2 \pi$. Thus, the resulting Vandermonde matrices are full column rank.

Let us denote by $\hat{\phi}_{q}$ the poles estimated with the HTLS method (see [5] for details). If the sampling period $T_{s}$ is chosen according to (8), then the angle of $\hat{\phi}_{q}$, denoted $\angle \hat{\phi}_{q}$, belongs to $[-\pi, \pi]$. We deduce the following estimator for the Doppler scaling factor:

$$
\hat{a}=-1+\frac{1}{K_{u}} \sum_{q=q_{0}}^{K_{u}+q_{0}-1} \frac{\angle \hat{\phi}_{q}}{2 \pi \varphi_{q} T_{e}} .
$$

One can note that such a Doppler scaling factor estimator is completely blind. However, since it is a SVD-based estimator, the computation load becomes significant when increasing $K_{u}$. So, we suggest to use few active subcarriers $K_{u}$. The choice of the optimal number is still an open question. Moreover, when dealing with subspace-based methods, the dimension of the initial matrix is very important. When applying HTLS, it has been shown that very good results are obtained for $L \times$ $N-L+1$ matrices where $L \in[N / 3,2 N / 3]$ [13].

\section{DATA DETECTION}

Now, for recovering the transmitted information symbols, we assume that some pilot symbols have been inserted in the second OFDM sub-block. We first estimates channel parameters using pilot symbols before recovering the informative symbols. Recall that the symbols $c_{q}$ are informative whereas only some of $d_{k}$ are also informative. The samples corresponding to the time interval $I_{2}$ are given by:

$$
y_{n}=\left.y(t)\right|_{t=n T_{e}}=\sum_{k=-K_{d} / 2}^{K_{d} / 2-1} d_{k} B_{k} e^{j 2 \pi(1+a) f_{k} n T_{e}} .
$$

In matrix form, we get:

$$
\mathbf{y}=\mathbf{H b}
$$

with $\mathbf{y}=\left(\begin{array}{lll}y_{n_{1}} & \cdots & y_{n_{1}+N_{1}-1}\end{array}\right)^{T}, \quad \mathbf{b}=$ $\left(\begin{array}{ccc}b_{-K_{d} / 2} & \cdots & b_{K_{d} / 2-1}\end{array}\right)^{T}, \quad b_{k}=d_{k} B_{k}, \quad \hat{\phi}_{k}=$ $e^{j 2 \pi(1+\hat{a}) f_{k} T_{e}}$, and

$$
\mathbf{H}=\left(\begin{array}{ccc}
\hat{\phi}_{-K_{d} / 2}^{n_{1}} & \cdots & \hat{\phi}_{K_{d} / 2-1}^{n_{1}} \\
\vdots & \ddots & \vdots \\
\hat{\phi}_{-K_{d} / 2}^{n_{1}+N_{1}-1} & \cdots & \hat{\phi}_{K_{d} / 2-1}^{n_{1}+N_{1}-1}
\end{array}\right) \in \mathbb{C}^{N_{1} \times K_{d}}
$$

It is straightforward to check that $\mathbf{H}$ is a full column rank matrix if $N_{1}>K_{d}$ and $T_{s} \leq \frac{1}{2 \lambda_{\max } \max \left\{f_{k}\right\}}$. As a 
consequence, we can compute the least squares (LS) solution of (10) as:

$$
\hat{\mathbf{b}}=\mathbf{H}^{\dagger} \mathbf{y}
$$

where $\mathbf{H}^{\dagger}$ denotes the pseudo-inverse operator of the matrix $\mathbf{H}$. Since the entries of $\hat{\mathbf{b}}$ result on the product of a symbol $d_{k}$ and the equivalent channel parameters $B_{k}$, without lack of generality, by assuming that the subcarriers numbered $k=$ $0,1, \cdots, K_{p}-1, K_{p}<K_{d} / 2$, are devoted to pilot symbols, i.e. $d_{k}, k=0,1, \cdots, K_{p}-1$, are known to the receiver, we get:

$$
\hat{B}_{k}=\hat{b}_{k} / d_{k} .
$$

Now, the question is: how constructing $\hat{B}_{k}$ associated with the information symbols from those estimated using the $K_{p}$ pilots?

First, we can note that (4) can also be written as

$$
B_{k}=\sum_{p=1}^{P} \gamma_{p} \zeta_{p}^{k}, \quad \gamma_{p}=A_{p} e^{-j 2 \pi f_{c} \tau_{p}}, \quad \zeta_{p}=e^{-j 2 \pi \Delta_{f} \tau_{p}}
$$

The expression above is particularly meaningful. Indeed, $B_{k}$ can be viewed as a mixture of exponentials in the frequency domain. We can therefore obtain both $\gamma_{p}$ and $\zeta_{p}$ by solving a harmonic retrieval problem.

Assuming that the number of significative paths $P$ is known and provided $K_{p} \geq 2 P$, we make use of the HTLS method for solving the problem (13) where $B_{k}$ is replaced by its estimated value $\hat{B}_{k}$. For this purpose, we build an $L \times M$ Hankel matrix B, with $K_{p}=L+M-1, L>P, M \geq P$, which admits the following Vandermonde decomposition:

$$
\mathbf{B}=\mathbf{S}_{2} \operatorname{diag}(\boldsymbol{\gamma}) \mathbf{T}_{2}^{T}
$$

with $\mathbf{S}_{\mathbf{2}}$ and $\mathbf{T}_{2}$ some Vandermonde matrices having $\zeta_{p}$ as generators, and $\gamma$ the vector with $\gamma_{p}$ as entries.

Let us denote $\hat{\zeta}_{p}$ the estimated poles. By replacing the poles by their estimated values in $\mathbf{S}_{2}$ and $\mathbf{T}_{2}$, we can solve the following equation:

$$
\operatorname{vec}(\mathbf{B})=\left(\mathbf{T}_{2} \odot \mathbf{S}_{2}\right) \boldsymbol{\gamma},
$$

$v e c($.$) denoting the vectorization operator whereas \odot$ stands for the Khatri-Rao product, i.e. a columnwise Kronecker product.

The entries of the least squares solution of (15), denoted $\hat{\gamma}$, can be used for computing $\hat{B}_{k}$ for any value of $k$ as $\hat{B}_{k}=$ $\sum_{p=1}^{P} \hat{\gamma}_{p} \hat{\zeta}_{p}^{k}$. Therefore, we can deduce the information symbols in the second sub-block as:

$$
\hat{d}_{k}=\frac{\hat{b}_{k}}{\sum_{p=1}^{P} \hat{\gamma}_{p} \hat{\zeta}_{p}^{k}} .
$$

while those of the first sub-block can be obtained as

$$
\hat{c}_{q}=\frac{\hat{\alpha}_{q}}{\eta e^{j 2 \pi \varphi_{q}(1+\hat{a}) n_{0} T_{s}} \sum_{p=1}^{P} \hat{\gamma}_{p} \hat{\zeta}_{p}^{q}} .
$$

$\hat{\alpha}_{q}$ being the corresponding entry of the least squares solution of the vectorized version of equation (7), i.e. $\operatorname{vec}(\mathbf{Y})=$ $\left(\mathbf{T}_{1} \odot \mathbf{S}_{1}\right) \boldsymbol{\alpha}$.

\section{Simulation Results}

In these simulations, the range of frequency used by the underwater vehicles was $[16 k H z-27 k H z]$. The Doppler scaling factor was set to $a=8 \times 10^{-4}$. We assume that $\left|a_{\max }\right|=10^{-3}$, meaning that the maximal relative speed is $1.5 \mathrm{~m} / \mathrm{s}$. The carrier frequency was set equal to $f_{c}=21$ $\mathrm{kHz}$, whereas the guard interval was $T_{g}=10 \mathrm{~ms}$. We used $K_{d}=256$ subcarriers for the second OFDM sub-block. The duration of this sub-block is $T_{d}=K_{d} / B$, with $B=11$ $\mathrm{kHz}$, whereas that of the first sub-block is $T_{u}=T_{d} / 10$. Hence, the duration of one OFDM block is $45.6 \mathrm{~ms}$. The data was modulated using a QPSK constellation. All the results presented below are averaged values over 100 independent Monte Carlo runs. The channel impulse response used for the simulation is depicted in figure 3. The additive noise was a complex valued white Gaussian noise.

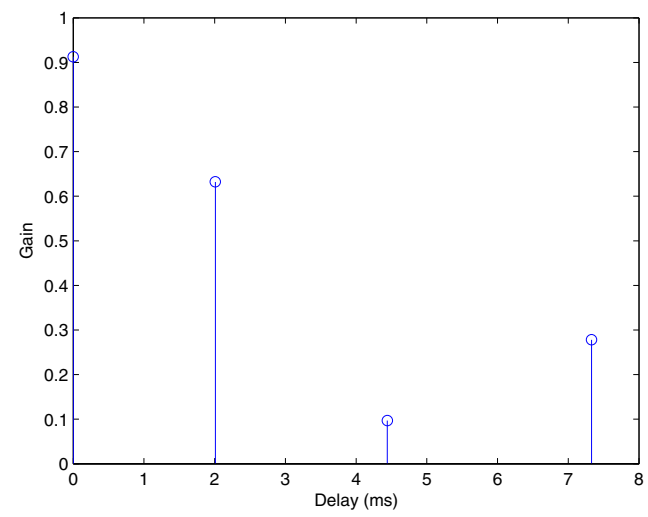

Fig. 3. Impulse response of the simulated channel

We evaluate the performance of the proposed scheme by computing the mean square error between the estimated and the actual values of the Doppler scaling factor. The data detection method is evaluated by means of the bit-error-rate (BER).

Fig. 4 depicts the mean value of the estimated Doppler scaling factor whereas the mean square error on the estimation of this factor is depicted in Fig. 5.

We can note the effectiveness of the Doppler estimation scheme. However, the performance is degraded when decreasing the SNR. The degradation is more accentuated when increasing the number of subcarriers in the first sub-block of the OFDM symbol. Indeed, by increasing $K_{u}$, the frequency spacing of the subcarriers is reduced. Therefore, the harmonic retrieval problem needs higher resolution and precision. Such a precision is difficult to obtain when the SNR decreases. Increasing $K_{u}$ seems to be relevant only for high SNR values. This observation is directly related to the behaviour of HTLS in noisy cases. 


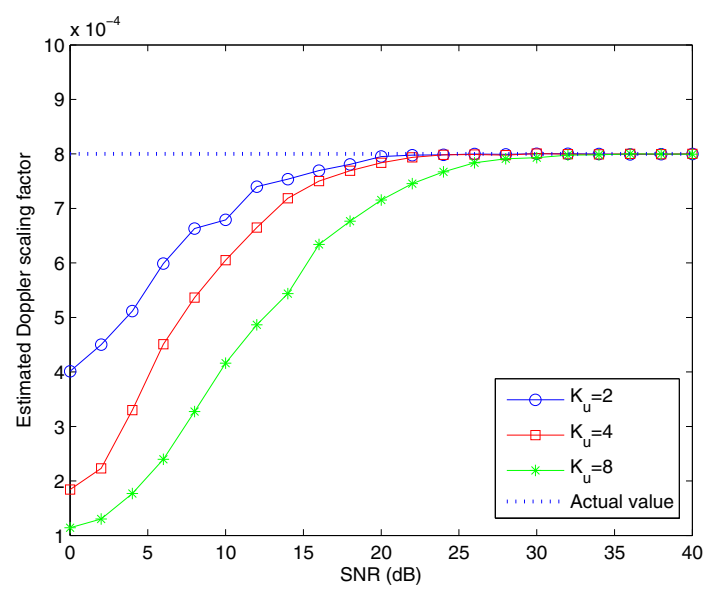

Fig. 4. Estimated value of the Doppler scaling factor.

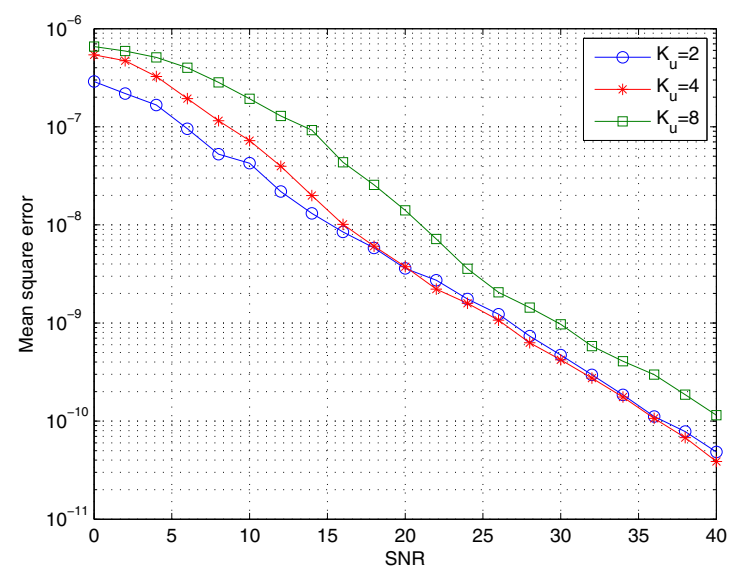

Fig. 5. Mean Square Error for the estimation of the Doppler scaling factor.

In Fig. 6, we depict the BER for three numbers $K_{p}$ of pilots and two values for $K_{u}$. Increasing the number of pilot allows improving the BER.

From these simulations, we can conclude that increasing the number of pilots has a positive effect on the performance of the receiver while increasing the number of subcarriers $K_{u}$ has a contrary effect.

\section{CONCLUSION}

In this paper, we have presented a new scheme for both estimating the Doppler scaling factor and detecting the informative data. In the proposed scheme, each OFDM symbol is divided in two parts. The first one is shorter and carries out few data with few subcarriers whereas the second one contains both informative and pilot symbols. The first sub-block serves to estimate the Doppler scaling factor while the pilots in the second sub-block are used for estimating the equivalent channel. Both estimation methods are based on high resolution methods for solving harmonic retrieval problems in time and frequency domains respectively. The proposed scheme is particularly efficient for moderate to high SNR. Robustness to noise, for lower levels of SNR, and extension to the multi-

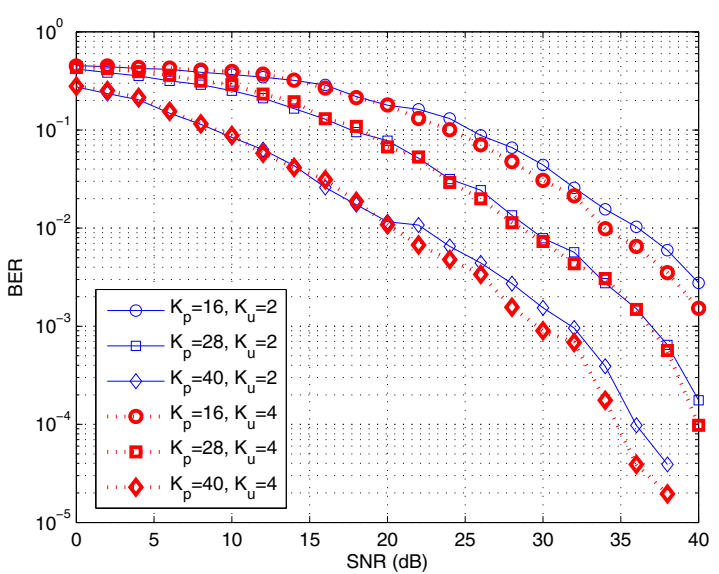

Fig. 6. BER for different number of pilots and two numbers of subcarriers in the first sub-block $\left(K_{d}=256\right)$

user case will be investigated. In addition, effectiveness of the proposed approach using experimental data is to be considered in future works.

\section{ACKNOWLEDGMENT}

This work was supported by the EU project FeedNetBack and the ANR project CONNECT.

\section{REFERENCES}

[1] B. Li, S. Zhou, M. Stojanovic, L. Freitag, and P. Willett, "Multicarrier communication over underwater acoustic channels with nonuniform doppler shifts," IEEE Journal of Oceanic Engineering, vol. 33, no. 2 pp. 198-209, 2008.

[2] A. Bahai and B. Saltzberg, Multi-carrier digital communications: theory and applications of OFDM. Springer, 1999.

[3] B. Sharif, J. Neasham, O. Hinton, and A. Adams, "A computationally efficient doppler compensation for underwater acoustic communications," IEEE Journal of oceanic engineering, vol. 25, no. 1, pp. 52-61, January 2000.

[4] B.-C. Kim and I.-T. Lu, "Parameter study of OFDM underwater communications system," in Proc. of OCEANS 2000 MTS/IEEE Conference and Exhibition, vol. 2, September 11-14 2000, pp. 1251-1255.

[5] A. Kibangou, C. Siclet, and L. Ros, "Joint channel and doppler estimation for multicarrier underwater communications," in Proc. of IEEE Int. Conf. on Acoustics, Speech, and Signal Processing (ICASSP) 2010, Dallas, Tx, USA, March 14-19 2010, pp. 5630-5633.

[6] —, "ZF OFDM receiver for underwater communications," in Proc. of Int. Symp. on Comm., Control and Signal Process. (ISCCSP) 2010 Limassol, Cyprus, March 3-5 2010.

[7] S. Kay, Modern spectral estimation, Theory and Application. Englewood Cliffs, New Jersey, USA: Prentice-Hall, 1988.

[8] P. Stoica and R. Moses, Introduction to spectral analysis. Englewood Cliffs, New Jersey, USA: Prentice-Hall, 1997.

[9] R. Roy and T. Kailath, "ESPRIT-Estimation of signal parameters via rotational invariance techniques," IEEE Trans. on Signal Proc., vol. 37, no. 7, pp. 984-995, July 1989.

[10] J. Papy, L. De Lathauwer, and S. Van Huffel, "Exponential data fitting using multilinear algebra: The single-channel and multi-channel case," Numer. Linear Algebra Appl., vol. 12, pp. 809-826, 2005.

[11] S. Van Huffel, "Enhanced resolution based on minimum variance estimation and exponential data modeling," Signal Processing, vol. 33, no. 3, pp. 333-355, 1993.

[12] S. Kung, K. Arun, and D. Bhaskar Rao, "State-space and singular value decomposition-based approximation methods for harmonic retrieval problem," Journal of the Optical Society of America, vol. 73, no. 12, pp. 1799-1811, 1983.

[13] S. Van Huffel, H. Chen, C. Decanniere, and P. Van Hecke, "Algorithm for time-domain NMR data fitting based on total least squares," Journal of Magnetic Resonance, vol. 110, no. series A, pp. 228-237, 1994. 